\title{
The role of flow cytometry in celiac disease screening using human leukocyte antigen in adult patients with type 1 diabetes mellitus
}

\author{
Miren Vicuña Arreguia, Jose Manuel Zozaya Urmeneta a , Helena León Brito ${ }^{b}$, Juan Pablo Martínez De Estebanc, \\ Carlos Prieto Martínez ${ }^{\mathrm{a}}$, Lluis Forga Llenas ${ }^{\mathrm{d}}$, Erkuden Aranburu Urtasune, Francisco Sala Pericase, \\ Ramón Angós Musgo', Maria Rosario Mercado Gutierrez ${ }^{\mathrm{g}}$, Mercedes Palacios Sarrasquetah \\ Complejo Hospitalario de Navarra, Pamplona; Hospital Reina Sofía, Tudela; Hospital García Orcoyen, Estella; Clínica \\ Universidad de Navarra, Pamplona, Spain
}

\section{Abstract}

\begin{abstract}
Background Patients with type 1 diabetes mellitus (DM1) have an increased risk of celiac disease (CD). Since CD can be seronegative, more sensible tests for detection are needed. In seronegative patients, CD diagnosis may be difficult because of a lack of specificity. Flow cytometry analysis of lymphocyte populations can be useful in this situation. We aimed to study the prevalence of CD in adult DM1 using human leukocyte antigen (HLA) compatibility-based screening. A secondary goal was to study the role of flow cytometry as a complementary tool in these patients.
\end{abstract}

Methods We selected 200 patients with DM1, of whom 190 (95\%) had HLA DQ2, DQ8 or both. Of these, 136 agreed to participate and provided epidemiological data. All patients underwent blood tests and gastroscopy.

Results Sixteen patients had a histology consistent with CD. After ruling out other diagnoses, 6 patients were diagnosed with CD, 2 of whom had negative antibodies. All were DQ2.5 homozygous, with a CD prevalence of $9.8 \%$ in this group. In the flow cytometry analysis of duodenal biopsy samples, when we compared all non-CD with CD patients, we found that the $\gamma / \delta$ intraepithelial lymphocyte (IEL) percentage was significantly higher and the CD3 negative IEL percentage significantly lower in the CD group. We found similar results when we compared only those with histological lesions.

Conclusions Screening of CD in patients with DM1 by HLA detects only $1 \%$ of seronegative patients with CD. DQ2.5 homozygous patients are at most risk of developing CD. The study of lymphocyte populations in the duodenal biopsy by flow cytometry discriminates patients with CD from those without CD with high sensitivity and specificity.

Keywords Celiac disease, flow cytometry, HLA typing, type 1 diabetes mellitus

Ann Gastroenterol 2017; 30 (1): 1-7
Departments of ${ }^{\mathrm{a}}$ Gastroenterology, Complejo Hospitalario de Navarra, Pamplona (Miren Vicuña Arregui, Jose Manuel Zozaya Urmeneta, Carlos Prieto Martínez); bastroenterology, Hospital Reina Sofía, Tudela


Orcoyen, Estella (Juan Pablo Martínez De Esteban); Endocrinology and Nutrition, Complejo Hospitalario de Navarra, Pamplona (Lluis Forga Llenas); ${ }^{\mathrm{e} H e m a t o l o g y, ~ C o m p l e j o ~ H o s p i t a l a r i o ~ d e ~ N a v a r r a, ~ P a m p l o n a ~}$ (Erkuden Aranburu Urtasun, Francisco Sala Pericas); ${ }^{\mathrm{f}}$ Gastroenterology, Clínica Universidad de Navarra, Pamplona (Ramón Angós

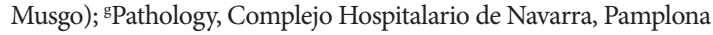

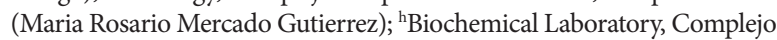
Hospitalario de Navarra, Pamplona (Mercedes Palacios Sarrasqueta), Spain

Conflict of Interest: None

Correspondence to: Dr. Miren Vicuña Arregui, Gastroenterology Department, Complejo Hospitaltario de Navarra, Irunlarrea 3, 31008 Pamplona, Spain, Tel.: +34 848422114, e-mail: Mirva77@hotmail.com

Received 26 July 2016; accepted 01 December 2016; published online 16 December 2016

DOI: https://doi.org/10.20524/aog.2016.0113

\section{Introduction}

Celiac disease $(\mathrm{CD})$ has an approximate prevalence of $1 \%$ worldwide [1-4]. This prevalence is greater in high-risk groups, including celiac patients' first-degree relatives and individuals with other diseases, such as type 1 diabetes mellitus (DM1) [5]. Celiac disease screening is recommended in highrisk groups [6] and is usually performed by serologic testing. However, these tests may not be sufficiently sensitive, especially during the initial stages of the histological alteration [7-9].

$\mathrm{CD}$ is only expressed in individuals with certain human leukocyte antigen (HLA) haplotypes [10]. Approximately 90-95\% of celiac patients are DQ2 carriers, also known as DQ2.5 (DQA1 $\left.{ }^{\star} 0501 / \mathrm{DQB1}{ }^{\star} 0201\right)$. Almost all of the remaining celiac patients express the DQ8 haplotype (DQA $1^{\star} 0301$ / DQB $\left.1^{\star} 0302\right)$. Only a very small percentage of patients express other genes or only one of the two alleles that make up the DQ2 haplotype [10]. Around 30\% of the general population 
express DQ2 and 20\% express DQ8 [11]. The high sensitivity of HLA typing has encouraged scientists to investigate its role in CD screening with the aim of diagnosing seronegative presentations. One study in which celiac patients' first-degree relatives were screened using HLA determination found that $\mathrm{CD}$ diagnosis could be improved significantly by applying this test compared to the most common strategy using serologic testing (20.8\% vs. $7.2 \%)$. In this study, the difference between the screening methods depended mostly on the diagnosis of patients at Marsh 1 stage [12].

On the other hand, $\mathrm{CD}$ diagnosis can be difficult to establish in patients with negative antibodies, because of the low specificity of the histological lesions. Flow cytometry may be useful in the study of the phenotypic characteristics of the duodenal intraepithelial lymphocyte (IEL) population. In CD, there is an increase in the total number of IELs and a relative increase in the proportion of IELs compared to the number of epithelial cells. Additionally, an increase in the percentage of $\gamma / \delta$ T-cell receptor (TCR) IELs and a decrease in the CD3-/ CD7+ IEL population has been described [13].

Considering all the above observations, we aimed to study the prevalence of CD in adult DM1 patients by performing screening based on HLA compatibility. In addition, we studied the role of flow cytometry as a complementary tool for the diagnosis of CD in this group of patients, defining the optimal cutoff values for $\gamma / \delta$ positive and CD3 negative IELs.

\section{Patients and methods}

\section{Study population}

This study was performed at a tertiary hospital with an area of influence of approximately 352,000 adult individuals. We initially evaluated 1056 patients with a diagnosis of DM1. Patients previously diagnosed with CD were excluded from our study.

It was calculated that a sample of 142 individuals would be needed to estimate the proportion of patients with CD among those diagnosed with DM1 (1056 DM1 patients, estimated CD prevalence of $4 \%$ in this group) with an accuracy of $\pm 3 \%$. Based on a previous study performed by our group [14], we considered a $70 \%$ participation acceptance rate; thus, we aimed to include 200 patients. These subjects were selected consecutively according to their order of attendance at the endocrinology consulting room. There were no first-degree relatives, twins or patients with latent autoimmune diabetes of adults.

\section{Study design}

Epidemiologic data, as well as antigliadin (AGA), antitissue transglutaminase (AtTG) and antiendomysium (EMA) antibody titers, total IgA levels and HLA typing results, were collected from the $200 \mathrm{DM} 1$ patients. In those patients with IgA deficiency, AtTG IgG class levels were ordered.
HLA-DQ2 or DQ8 positive patients were assessed at an outpatient visit and were invited to have an upper gastrointestinal endoscopy (UGIE). In those patients who consented, 5 distal duodenum biopsies were taken, 2 from the gastric antrum and 2 from the gastric corpus. Of the 5 duodenal biopsies, 1 was collected in saline solution for the study of the lymphocytic population with flow cytometry and the other 4 remaining samples were collected in formaldehyde solution for histological examination. Patients with histological findings that suggested CD were contacted and seen again in the gastroenterology consulting room.

\section{Antibody determination}

EMA were quantified by indirect immunofluorescence on sections from the distal portion of monkey esophagus as an antigenic substrate (MeDiCa Diagnostics, Encinitas, CA, USA). Sera were diluted at a 1:5 concentration in a $50 \mathrm{mM}$ and pH 7.8 phosphate buffer. Concentrations above these dilution values were considered as positive.

AGA were quantified by an immunoenzymatic method, using a commercial kit with gliadin extracted from wheat as a substrate (Orgentec Diagnostika, GMBH, Mainz-Germany). Values over 10 arbitrary units were considered as positive.

AtTG were assessed, following the manufacturer's instructions, using an immunoenzymatic method that uses human recombinant transglutaminase as a substrate (Orgentec Diagnostika, GMBH, Mainz-Germany). Values above $15 \mathrm{U} / \mathrm{mL}$ were considered as positive.

\section{HLA determination}

Blood samples that had been anticoagulated with ethylenediaminetetraacetic acid (EDTA) were obtained from each patient. DNA extraction was achieved using paramagnetic particles (Maxwell ${ }^{\circledR} 16$ System). DNA concentrations for each sample were measured by spectrophotometry (NanoDrop ${ }^{\oplus}$ ) and further concentration adjustments were performed in order to study DRB1 and DQB1 loci with a low resolution PCR-SSO technique (sequence-specific oligonucleotides; Luminex ${ }^{\oplus}$ ). Samples identified as $\mathrm{DRB}^{\star} 03 / \mathrm{DQB} 1^{\star} 02$ or $\mathrm{DRB} 1^{\star} 04 / \mathrm{DQB} 1^{\star} 03$ were subsequently studied for the allelic assignation, including the DQA1 locus, using a high resolution polymerase chain reaction technique with sequence specific primers (PCR-SSP). Results were presented as a serological annotation in which patients were DQ2 and/or DQ8 either positive or negative.

\section{Histological examination}

Four distal duodenum biopsies and four gastric biopsies were collected for the histological examination. Biopsy material was fixed in a $4 \%$ formaldehyde dilution and embedded in paraffin. Three $4-\mu \mathrm{m}$ thick slices were later obtained for each sample and 
hematoxylin-eosin stain was applied. Gastric antral biopsies were additionally stained with Giemsa. All of the samples were studied by the same expert pathologist. Duodenal lesions, if any, were classified following Oberhuber's [15] modification of Marsh's criteria [16].

\section{Lymphocytic population study}

One of the duodenal biopsies was collected in a saline solution and was incubated in culture medium, with the addition of $50 \mu \mathrm{L}$ EDTA $+50 \mu \mathrm{L}$ dithiothreitol $(0.1 \mathrm{M})$, for 60-90 min at room temperature under moderate stirring using a vertical rotor. Once the cell suspensions were obtained, the mixture was centrifuged for $5 \mathrm{~min}$ at $1500 \mathrm{rpm}$ (no brake). The supernatant was then decanted and the sample was resuspended in $1 \mathrm{~mL}$ of culture medium. Culture medium was prepared with $10 \mathrm{~mL}$ of fetal serum (hemotherapy), $88 \mathrm{~mL}$ of culture medium, $1 \mathrm{~mL}$ of antibiotic and $1 \mathrm{~mL}$ of glutamine.

Immunophenotyping was performed using one tube per sample and a control tube. The following monoclonal antibodies, tagged with different fluorochromes, were added to $200 \mu \mathrm{L}$ of cellular suspension: $10 \mu \mathrm{L}$ of anti-CD103fluorescein isocyanate, $10 \mu \mathrm{L}$ of anti-TCR $\gamma \delta$-phycoerythrin (PE), $5 \mu \mathrm{L}$ of anti-CD3-PE- cyanine 7 , and $5 \mu \mathrm{L}$ of anti-CD45allophycocyanin (Becton, Dickinson [BD] and Company). After 30 min incubation at $4^{\circ} \mathrm{C}$ in a dark box, cells were washed with phosphate buffered saline (PBS) for $7 \mathrm{~min}$ at $1200 \mathrm{rpm}$ (no brake) and the sample was decanted; $500 \mu \mathrm{L}$ of PBS were then added to each tube.

Flow cytometry analysis was performed using BD's FACSCalibur $^{\mathrm{TM}}$ cytometer. Forward and side scatter signals (FSC, SSC) were recorded on a linear scale, whereas fluorescence signals were registered on a logarithmic scale. Multiparametric data were collected using a scatter threshold that excluded erythroid cells and cellular debris. Lymphocytes were selected using a lymphocyte selection gate based on a SSC/CD 45 (light dispersion at $90^{\circ}$ ) histogram. A negative isotopic control was applied in order to adjust the fluorescence intensities. Data were analyzed using BD's CellQuest ${ }^{\mathrm{TM}}$ program.

\section{CD diagnosis}

Patients were classified as having possible $\mathrm{CD}$ when they had histological lesions suggestive of CD and all other pathological entities with similar histological changes had been ruled out. Thus, taking into account the high specificity of the antibodies, patients who had histological changes suggestive of $\mathrm{CD}$ and positive antibodies were considered as possible CD cases.

Special attention was paid to excluding other possible diagnoses in Marsh 1 and 2 type cases with CD negative antibodies. Thus, as well as considering patients' personal history and clinical records, we asked individuals with Marsh 1 and 2 type lesions and negative antibodies about their use of nonsteroid anti-inflammatory drugs (NSAIDs) in the previous month and tested them for Helicobacter pylori $(H P)$. Those patients with a positive $H P$ test received eradication therapy and had a new UGIE with duodenal biopsies at least 6 months after treatment was completed, to evaluate the presence of histological changes. None of the patients with Marsh 1 type changes had taken NSAIDs in the month before the endoscopy was performed. Those patients who were HP negative, with no NSAID consumption and without any symptoms, were followed at the outpatient clinic and underwent an additional UGIE with duodenal biopsies at 2 years to assess whether histological lesions persisted or had spontaneously reverted. Cases in which histological changes persisted either after $H P$ eradication therapy or after 2-year follow up were classified as possible CD.

Patients with Marsh 2 type lesions and negative antibodies were asked about previous NSAID consumption and their HP status was assessed. Again, none of the patients in this group had taken NSAIDs during the month before the endoscopy was performed. Those cases that were found to be positive for $H P$ were treated in the same way as patients with lymphocytic enteritis. HP negative patients and those without a history of NSAID consumption were classified as possible CD cases.

All of the patients with a "possible CD" diagnosis were advised to follow a gluten-free diet (GFD). Definite CD diagnosis was made in those cases with a symptomatic improvement (when symptoms had been present) and a serological negativization (when antibodies had been positive) or a histological improvement (when antibodies had been negative) after at least 1 year on a GFD.

\section{GFD compliance follow up}

GFD compliance was assessed by a detailed anamnesis during the subsequent interviews. Serological response (antibody negativization) was evaluated in those patients with positive antibodies at the beginning of the study. In those cases with negative antibodies, histological response was studied by performing an UGIE with duodenal biopsies after two years.

\section{Statistical analysis}

Study variables were recorded in a database that was set up with the IBM SPSS Statistics 15.0 program (Chicago, USA). Comparisons were performed with two-tailed tests. P-values less than 0.05 were considered statistically significant. A normal distribution could not be assumed for the CD patients' group, because of the small number of individuals with this diagnosis. Therefore, comparisons related to lymphocytic populations between $\mathrm{CD}$ and non-CD patients were performed using the Mann-Whitney $U$ test. The predictive power of flow cytometry was calculated with a receiver operating characteristic (ROC) curve, taking the value at which the sum of the sensitivity and specificity was the highest as the optimal cutoff point. 


\section{Results}

\section{Epidemiological characteristics}

Of the 200 DM1 patients initially evaluated in our study, 87 (43.5\%) were female and 113 (56.5\%) were male. The median age of our study population was 41 years (range: 18-83).

One hundred ninety patients (95\%) were either HLA DQ2 or DQ8 positive. The HLA distribution is shown in Fig. 1. Nineteen patients $(9.5 \%)$ were positive for at least one of the three antibodies that were tested in our study. All of the patients with positive antibodies were HLA DQ2 or DQ8 positive. The 190 DM1 patients who were HLA DQ2 or DQ8 positive were invited to complete a gastrointestinal study for $\mathrm{CD}$ detection. One hundred thirty-six patients finally accepted.

Fifty-two (38.2\%) of the 136 patients who underwent surveillance were female and $84(61.8 \%)$ were male. The median age of the patients in this group was 41.5 years (range 18-74).
Median age at DM1 diagnosis in this group was 24.5 years (range: 1-64); the median age of patients with a two-year DM1 progression was 15.5 years (range $0-48$ ). HLA distribution is shown in Fig. 1.

\section{CD prevalence}

Of the 136 patients studied, 16 had histological findings that suggested CD. Ten of them were at initial stages (Marsh 1 and 2 type lesions) and 6 had Marsh 3 type lesions (Fig. 2). Table 1 shows the diagnostic sequence followed. Patient number 9 had a previous history of common variable immunodeficiency. This entity can cause an enteropathy that is similar to $\mathrm{CD}$, and some patients show a histological response after a GFD [17]. Thus, patient number 9 was encouraged to follow a GFD for 2 years, after which a new histological assessment was performed. After 2 years on a strict GFD, the histological changes were similar to those at baseline endoscopy, so a diagnosis of CD could not be

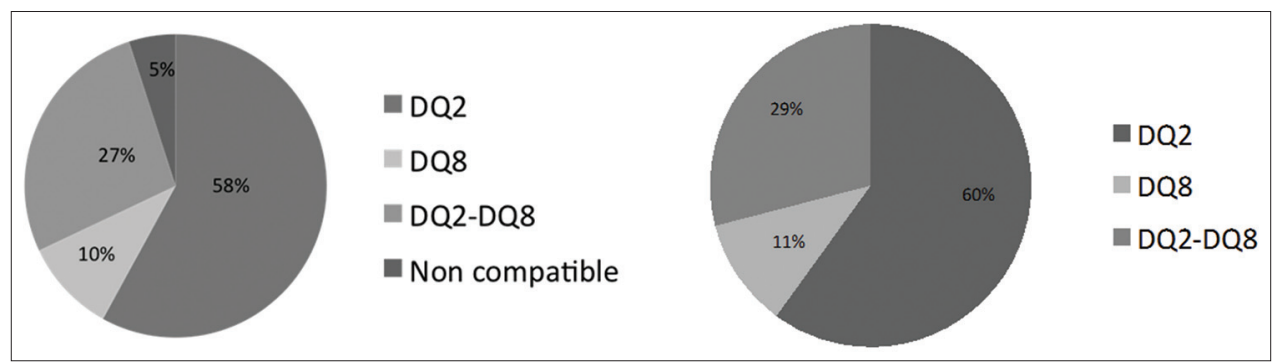

Figure 1 Distribution of human leukocyte antigen in our initially evaluated population and in patients who consented to participate in the study

Table 1 Patients with histological findings that suggested CD

\begin{tabular}{|c|c|c|c|c|c|c|}
\hline Patients & Histology & Antibodies & $H P$ & Follow up & Control & $\mathrm{CD}$ \\
\hline 1 & Marsh 1 & $(+)$ & & GFD & Negative $A b$ & Yes \\
\hline 2 & Marsh 1 & $(-)$ & $(+)$ & $H P$ eradication & Normal biopsy & No \\
\hline 3 & Marsh 1 & $(-)$ & $(+)$ & $H P$ eradication & Normal biopsy & No \\
\hline 4 & Marsh 1 & $(-)$ & $(+)$ & $H P$ eradication & No control & \\
\hline 5 & Marsh 1 & $(-)$ & $(-)$ & & Normal biopsy & No \\
\hline 6 & Marsh 1 & $(-)$ & $(-)$ & & Normal biopsy & No \\
\hline 7 & Marsh 1 & $(-)$ & $(-)$ & & No control & \\
\hline 8 & Marsh 2 & $(-)$ & $(+)$ & $H P$ eradication & No control & \\
\hline 9 & Marsh 2 & $(-)$ & $(-)$ & GFD (CVI) & Marsh 2 & No \\
\hline 10 & Marsh 2 & $(-)$ & $(-)$ & GFD & Normal biopsy & Yes \\
\hline 11 & Marsh 3a & $(+)$ & & GFD & Negative $A b$ & Yes \\
\hline 12 & Marsh 3a & $(-)$ & & No GFD & Normal biopsy & No \\
\hline 13 & Marsh 3a & $(-)$ & & No GFD & Normal biopsy & No \\
\hline 14 & Marsh 3a & $(-)$ & & GFD & Normal biopsy & Yes \\
\hline 15 & Marsh 3c & $(+)$ & & GFD & Negative $A b$ & Yes \\
\hline 16 & Marsh 3c & $(+)$ & & GFD & Negative $A b$ & Yes \\
\hline
\end{tabular}

CD, celiac disease; GFD, gluten-free diet; Ab, antibodies; CVI, common variable immunodeficiency; HP, Helicobacter pylori 
made. Patient number 12 had recently been admitted with septic shock, and did not receive the recommendation to follow a GFD initially. A new examination was performed after 1 year, showing a normalization of the duodenal biopsies; CD diagnosis was therefore ruled out. Even though patient number 13 acknowledged frequent dietary transgressions, a control UGIE yielded a normal duodenal biopsy. Because the histological improvement could not be explained by the GFD, this patient was not diagnosed with definite CD.

In total, 6 patients were diagnosed with definite CD. This comprised $3 \%$ of the initially selected patients and $4.4 \%$ of the cases who underwent a complete study. The patient distribution is represented in Fig. 3. All CD patients were positive for HLADQ2.

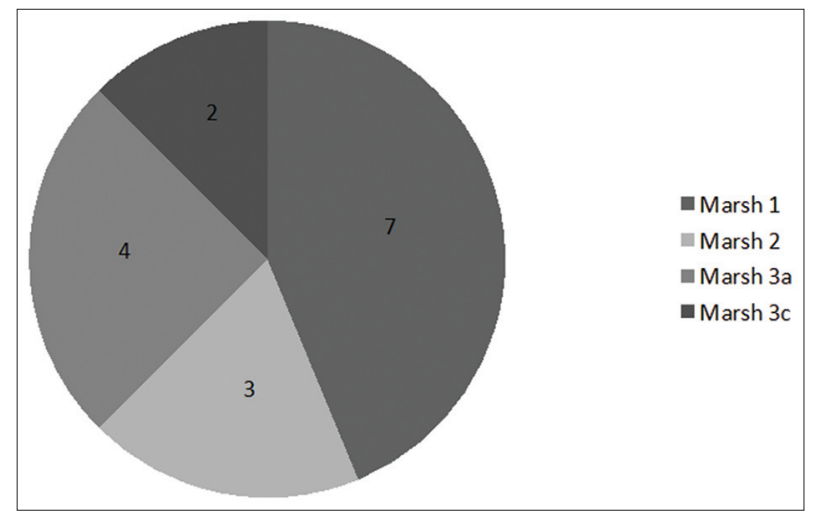

Figure 2 Distribution of histological findings compatible with celiac disease



Figure 3 Patients with a definite diagnosis of celiac disease Ab, antibodies

\section{High resolution PCR molecular analysis of HLA results}

Once our study was completed, we analyzed whether certain HLA haplotypes were related to the risk of developing CD. Fig. 4 shows the results of the HLA analysis with molecular biology techniques using high resolution PCR. All patients (100\%) diagnosed with CD were homozygous for DQ2.5/DQ2.5. Taking our initial sample, the prevalence of CD in DQ2.5/DQ2.5 homozygous patients was $9.2 \%$ (6 of 65 cases).

\section{Lymphocytic population study in duodenal biopsies}

\section{Comparison between all of the patients with and without $C D$ diagnosis}

Table 2 shows the results of the duodenal biopsy flow cytometry analysis. The optimal cutoff values found in the ROC curves in order to distinguish healthy subjects from CD patients were:

- $\gamma / \delta$ IEL: values under $17 \gamma / \delta$ IELs per 100 were considered as normal. $\mathrm{AUC}=0.96$ (95\% CI 0.91-1)

- CD3 negative IEL: values over 5 CD3 negative IELs per 100 were considered as normal. AUC $=0.83$ (95\% CI 0.72-0.94)

\section{Comparison between patients with and without $C D$ presenting histological lesions}

When only the patients with histological findings compatible with CD were studied, a comparison of the IEL distribution in definite $\mathrm{CD}$ patients with the distribution in subjects with lesions in whom CD was finally ruled out revealed no significant differences in the percentage of total IELs between the 2 groups. However, in the group of CD patients the $\gamma / \delta$ IEL proportion was found to be significantly higher and the CD3 negative IEL proportion significantly lower (Table 3).

When assessing the optimal cutoff values for distinguishing among patients with $\mathrm{CD}$ compatible histological changes, those with and without definite $\mathrm{CD}$, we found that:

- $\quad \gamma / \delta$ IEL: values under $13.5 \gamma / \delta$ IELs per 100 were considered as normal. AUC $=1$ (95\% CI 1-1).

- CD3 negative IEL: values over 5 CD3 negative IELs per 100 were considered as normal. AUC $=0.88$ (95\% CI 0.71-1).

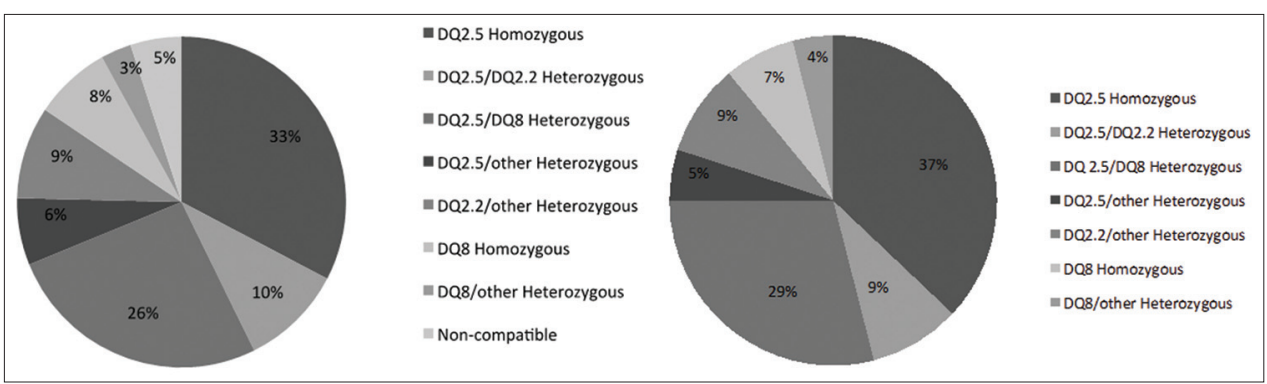

Figure 4 Distribution of human leukocyte antigen in our initially evaluated population of 200 patients and in patients who consented to participate in the study 
Table 2 Lymphocytic population in duodenal biopsies, comparing CD patients with DM1 non-CD patients

\begin{tabular}{lccc}
\hline Lymphocytic population & Non-CD & CD & P value \\
\hline Total lymphocyte \% & $2.1(0.5-10)$ & $2.5(0.9-3.8)$ & 0.89 \\
$\gamma / \delta$ lymphocyte \% & $6.8(1.7-31)$ & $23.8(17.3-48.5)$ & $<0.001$ \\
CD3-lymphocyte \% & $10.8(0.1-35.9)$ & $3.2(0.7-8.9)$ & 0.007 \\
\hline
\end{tabular}

Results are expressed as median values (range). Total lymphocyte \%: number of IEL per 100 epithelial cells. $\gamma / \delta$ IEL \%: number of $\gamma / \delta$ IEL per 100 IEL. CD3- IEL \%: number of CD3- IEL per 100 IEL. CD, celiac disease; DM1, type 1 diabetes mellitus, IEL, intraepithelial lymphocytes

Table 3 Lymphocytic population in duodenal biopsies, comparing CD patients with DM1 patients presenting histological impairment in which CD diagnosis was finally ruled out

\begin{tabular}{lccc}
\hline Lymphocytic population & Non-CD & CD & P value \\
\hline Total lymphocyte \% & $3.9(1-10)$ & $2.5(0.9-3.8)$ & 0.192 \\
$\gamma / \delta$ lymphocyte \% & $4.5(2.4-9.8)$ & $23.8(17.3-48.5)$ & 0.001 \\
CD3-lymphocyte \% & $8(1-26.7)$ & $3.2(0.7-8.9)$ & 0.013 \\
\hline
\end{tabular}

Results are expressed as median values (range). Total lymphocyte \%: number of IEL per 100 epithelial cells. $\gamma / \delta$ IEL \%: number of $\gamma / \delta$ IEL per 100 IEL. CD3- IEL \%: number of CD3- IEL per 100 IEL. CD, celiac disease;

$D M 1$, type 1 diabetes mellitus, IEL, intraepithelial lymphocytes

\section{Discussion}

Patients in the risk groups for developing $\mathrm{CD}$ can take advantage of CD diagnosis screening programs [6]. Screening has been performed in these risk groups traditionally by antibody (AtTG and EMA) testing. However, this strategy may fail in the detection of CD patients with negative antibodies. Therefore, alternative screening strategies are needed.

HLA typing has a high negative predictive value that permits, almost completely, the exclusion of patients who are not at risk of developing CD. HLA typing has been found to be helpful in screening for CD in first-degree relatives of CD patients, even in cases in which antibodies are negative [12]. We aimed to assess whether HLA typing could also be a suitable strategy for $\mathrm{CD}$ screening in DM1 patients. To date, the studies that have tested the role of HLA typing in DM1 cases and in CD and DM1 patients have been case-control studies in which the prevalence of the different HLA haplotypes was recorded in each group. In these studies, the proportion of DM1 HLA-DQ2.5 positive patients was lower (53-59\%) than the prevalence in our series, and similar to the prevalence that has been found in CD patients' first-degree relatives [18,19]. As we expected the prevalence of HLA-DQ2 positive cases among DM1 patients to be similar to the rate of HLA-DQ2 positivity in first-degree relatives, we considered the possibility of following the same screening strategy in this risk group for our study.

Using HLA typing as a screening strategy in DM1 patients allowed us to diagnose 6 cases with definite CD, which represented a $3 \%$ prevalence of $\mathrm{CD}$ in this group. Even though this prevalence is 3 times higher than that found in the general population, it is similar to the one found by our group and other researchers when using antibody testing for CD screening only $[14,20,21]$. Therefore, HLA typing in DM1 cases does not seem to have an additional value for the screening of CD in these patients. Since 4 of the 6 patients diagnosed with definite CD showed positive antibodies, HLA typing in our study was only useful in diagnosing 2 patients with CD and negative serology. It should be noted that our DM1 patient sample came from the same population as the patient sample from our previous study, in which CD screening was performed by measuring antibodies [14]; thus previously diagnosed CD patients were excluded. This might therefore mean that the prevalence of CD in our DM1 population was slightly greater than the prevalence that has been estimated in each of our studies separately.

Our findings do not agree with the results from the studies including first-degree relatives, in which screening using HLA typing increased CD detection from $7.2 \%$, when only serological analysis was performed, to $20.8 \%$ [12]. This difference between screening tests is explained by the higher diagnostic yield of HLA as compared to antibody testing for Marsh 1 type lesions, in which serological analysis has shown a lower sensitivity. However, other causes of lymphocytic enteritis were not ruled out and, in consequence, CD prevalence and the role of HLA typing for its detection might have been overestimated. If we had followed the same approach in our study, 6 patients with Marsh 1 type lesions, 2 patients with Marsh 2 type lesions and 2 patients with Marsh 3 type lesions would have been incorrectly diagnosed with CD. Thus, an increase in CD prevalence would have been found from $2 \%$, when testing antibodies only, to $8 \%$, when determining HLA.

Although more studies are probably needed in order to establish the role of HLA typing as a screening test for CD in risk populations, the 2012 guidelines of the European Society for Pediatric Gastroenterology, Hepatology, and Nutrition introduced HLA typing for the selection of highrisk patients who should subsequently undergo serological determinations [22]. On the other hand, considering that different HLA haplotypes entail different risks for CD, a possible approach could include variable intervals for repeating antigen determination based on HLA subtypes. In our study, all of the patients who were diagnosed with CD were DQ2.5/ DQ2.5 homozygous, with a $9.2 \%$ prevalence of CD in this group. Taking into account our results, a closer follow up in patients with this HLA phenotype seems reasonable.

Lymphocytic population study of duodenal biopsies using flow cytometry is an additional approach for the diagnosis of $\mathrm{CD}$ that is being assessed nowadays [13-23]. In our study, we found that celiac DM1 patients have an increased proportion of $\gamma / \delta$ IELs and a lower percentage of CD3 negative IELs as compared to non-celiac DM1 patients. The calculated optimal cutoff values in our trial, both with an area under the curve (AUC) greater than 0.8 , are similar to those that have been described by other groups [13]. Flow cytometry was also selective when comparing patients with definite CD to cases with CD-like histological impairment, showing similar cutoff points and AUC values. In the light of our findings, flow cytometry could be helpful in avoiding one of the pitfalls of the screening programs by distinguishing which patients, out 


\section{Summary Box}

\section{What is already known:}

- Patients with type 1 diabetes mellitus have an increased risk of celiac disease (CD), for which screening is indicated

- CD screening based on serological methods will fail to diagnose a percentage of celiac patients, because of seronegative forms

- Since CD histology has low specificity, seronegative forms may be difficult to diagnose

\section{What the new findings are:}

- We cannot recommend HLA-based CD screening because it only detects a small percentage of seronegative $\mathrm{CD}$ patients

- HLA typing can help identifying different risk groups for CD

- Flow cytometry analysis of lymphocyte populations is a very sensitive and specific tool for the diagnostic approach to CD, especially in unclear cases

of the group with negative antibodies and mild CD-compatible histology, have definite CD.

In the light of our results, to date, we cannot recommend HLA typing for the screening of CD in DM1 adult patients. Nevertheless, HLA can play an important role in identifying different risk groups for developing $\mathrm{CD}$, thus leading to tailored risk-based screening strategies. Lymphocytic population study of duodenal biopsies with flow cytometry could be an additional tool for the diagnosis of $\mathrm{CD}$, especially in unclear cases with a CD-compatible histology.

An optimal diagnostic algorithm has not yet been developed for the early detection of CD in this group of patients. Therefore, further investigations in this direction are needed.

\section{References}

1. Dubé C, Rostom A, Sy R, et al. The prevalence of celiac disease in average-risk and at-risk Western European populations: a systematic review. Gastroenterology 2005;128:S57-S67.

2. West J, Logan RF, Hill PG, et al. Seroprevalence, correlates, and characteristics of undetected coeliac disease in England. Gut 2003;52:960-965.

3. Mäki M, Mustalahti K, Kokkonen J, et al. Prevalence of Celiac disease among children in Finland. $N$ Engl $\mathrm{J} \mathrm{Med}$ 2003;348:2517-2524.

4. Fasano A, Berti I, Gerarduzzi T, et al. Prevalence of celiac disease in at-risk and not-at-risk groups in the United States: a large multicenter study. Arch Intern Med 2003;163:286-292.

5. Fernández-Bañares F, Esteve-Comas M, Rosinach M. [Screening for celiac disease in high risk groups]. Gastroenterol Hepatol 2005;28:561-566. [Spanish]

6. Mariné Guillem M, Esteve Comas M. [How to approach screening for celiac disease in 2008]. Gastroenterol Hepatol 2008;31:454-458. [Spanish]

7. Tursi A, Brandimarte G, Giorgetti G, Gigliobianco A, Lombardi D, Gasbarrini G. Low prevalence of antigliadin and anti-endomysium antibodies in subclinical/silent celiac disease. Am J Gastroenterol 2001;96:1507-1510.

8. Abrams JA, Diamond B, Rotterdam H, Green PH. Seronegative celiac disease: increased prevalence with lesser degrees of villous atrophy. Dig Dis Sci 2004;49:546-550.

9. Tursi A, Brandimarte G, Giorgetti GM. Prevalence of antitissue transglutaminase antibodies in different degrees of intestinal damage in celiac disease. J Clin Gastroenterol 2003;36:219-221.

10. Karell K, Louka AS, Moodie SJ, et al; European Genetics Cluster on Celiac Disease. HLA types in celiac disease patients not carrying the $\mathrm{DQA1}^{*} 05-\mathrm{DQB} 1{ }^{*} 02$ (DQ2) heterodimer: results from the European Genetics Cluster on Celiac Disease. Hum Immunol 2003;64:469-477.

11. Cassinotti A, Birindelli S, Clerici M, et al. HLA and autoimmune digestive disease: a clinically oriented review for gastroenterologists. Am J Gastroenterol 2009; 104:195-217.

12. Esteve M, Rosinach M, Fernández-Bañares F, et al. Spectrum of gluten-sensitive enteropathy in first-degree relatives of patients with coeliac disease: clinical relevance of lymphocytic enteritis. Gut 2006;55:1739-1745.

13. Olivencia Palomar P, Cano Ruiz A, Martín Scapa MA, León Prieto F, Roy Ariño G, Redondo Verge C. [Adult celiac disease and intraepithelial lymphocytes. New options for diagnosis?] Gastroenterol Hepatol 2008;31:555-559. [Spanish]

14. Vicuña Arregui M, Zozaya Urmeneta JM, Martínez de Esteban JP, et al. [Study of celiac disease in adults with type 1 diabetes mellitus]. Gastroenterol Hepatol 2010;33:6-11. [Spanish]

15. Oberhuber G, Granditsch G, Vogelsang H. The histopathology of coeliac disease: time for a standardized report scheme for pathologists. Eur J Gastroenterol Hepatol 1999;11:1185-1194.

16. Marsh MN. Gluten, major histocompatibility complex, and the small intestine. A molecular and immunobiologic approach to the spectrum of gluten sensitivity ('celiac sprue'). Gastroenterology 1992;102:330-354.

17. Malamut G, Verkarre V, Suarez F, et al. The enteropathy associated with common variable immunodeficiency: the delineated frontiers with celiac disease. Am J Gastroenterol 2010;105:2262-2275.

18. Doolan A, Donaghue K, Fairchild J, Wong M, Williams AJ. Use of HLA typing in diagnosing celiac disease in patients with type 1 diabetes. Diabetes Care 2005;28:806-809.

19. Sumnik Z, Cinek O, Bratanic N, et al. Risk of celiac disease in children with type 1 diabetes is modified by positivity for HLA-DQB $1^{\star} 02-\mathrm{DQA1}{ }^{\star} 05$ and TNF $-308 \mathrm{~A}$. Diabetes Care 2006;29:858-863.

20. Goh C, Banerjee K. Prevalence of coeliac disease in children and adolescents with type 1 diabetes mellitus in a clinic based population. Postgrad Med J 2007;83:132-136.

21. Barera G, Bonfanti R, Viscardi M, et al. Occurrence of celiac disease after onset of type 1 diabetes: a 6-year prospective longitudinal study. Pediatrics 2002;109:833-838.

22. Husby S, Koletzko S, Korponay-Szabó IR, et al; European Society for Pediatric Gastroenterology, Hepatology, and Nutrition. European Society for Pediatric Gastroenterology, Hepatology, and Nutrition guidelines for the diagnosis of coeliac disease. J Pediatr Gastroenterol Nutr 2012;54:136-160.

23. Calleja S, Vivas S, Santiuste M, et al. Dynamics of non-conventional intraepithelial lymphocytes-NK, NKT, and $\gamma \delta$ T-in celiac disease: relationship with age, diet, and histopathology. Dig Dis Sci 2011;56:2042-2049. 